\title{
Dynamic Interaction between P-Bodies and Transport Ribonucleoprotein Particles in Dendrites of Mature Hippocampal Neurons
}

\author{
Manuel Zeitelhofer, ${ }^{1}$ Daniela Karra, ${ }^{1}$ Paolo Macchi, ${ }^{1,2}$ Marco Tolino, ${ }^{1}$ Sabine Thomas, ${ }^{1}$ Martina Schwarz, ${ }^{1}$ \\ Michael Kiebler, ${ }^{1}$ and Ralf Dahm ${ }^{1}$ \\ ${ }^{1}$ Department of Neuronal Cell Biology, Center for Brain Research, Medical University of Vienna, A-1090 Vienna, Austria, and ${ }^{2}$ Centre for Integrative \\ Biology, Laboratory of Molecular and Cellular Neurobiology, University of Trento, 38060 Mattarello, Trento, Italy
}

\begin{abstract}
The dendritic localization of mRNAs and their subsequent translation at stimulated synapses contributes to the experience-dependent remodeling of synapses and thereby to the establishment of long-term memory. Localized mRNAs are transported in a translationally silent manner to distal dendrites in specific ribonucleoprotein particles (RNPs), termed transport RNPs. A recent study suggested that processing bodies (P-bodies), which have recently been identified as sites of RNA degradation and translational control in eukaryotic cells, may participate in the translational control of dendritically localized mRNAs in Drosophila neurons. This study raised the interesting question of whether dendritic transport RNPs are distinct from P-bodies or whether those structures share significant overlap in their molecular composition in mammalian neurons. Here, we show that P-body and transport RNP markers do not colocalize and are not transported together in the same particles in dendrites of mammalian neurons. Detailed time-lapse videomicroscopy analyses reveal, however, that both P-bodies and transport RNPs can interact in a dynamic manner via docking. Docking is a frequent event involving as much as $50 \%$ of all dendritic P-bodies. Chemically induced neuronal activity results in a $60 \%$ decrease in the number of P-bodies in dendrites, suggesting that P-bodies disassemble after synaptic stimulation. Our data lend support to the exciting hypothesis that dendritically localized mRNAs might be stored in P-bodies and be released and possibly translated when synapses become activated.
\end{abstract}

Key words: P-bodies; ribonucleoprotein particles (RNPs); hippocampal neurons; dendritic localization; dynamic movement; RNA localization

\section{Introduction}

The localization of mRNAs is an efficient way to target gene products to specific regions in a cell and occurs in a wide range of organisms and cell types (St Johnston, 2005; Bramham and Wells, 2007). In highly polarized cells, such as in neurons, the dendritic localization of mRNAs and their subsequent translation at stimulated synapses is believed to contribute to the experience-

Received Jan. 10, 2008; revised May 15, 2008; accepted May 25, 2008.

This work was supported by the Schram-Foundation, the Hochschuljubiläumsstiftung of the City of Vienna, and the European Science Foundation Eurocores Program "RNAQuality" (all to M.K.) and by Austrian Science Funds to P.M. and M.K. We are very grateful to Dr. P. Pasierbek (Institute of Molecular Pathology-Institute of Molecular Biotechnology, Vienna, Austria) for help with deconvolution and confocal microscopy. We thank the following people for reagents and/or advice: Drs. H. Blau (Stanford University, Palo Alto, CA), S. Hüttelmaier (Zentrum für Angewandte Medizinische und Humanbiologische Forschung, Halle, Germany), M. Kunze (Medical University of Vienna, Vienna, Austria), G. Meister (MPI, Martinsried, Germany), M. Ramaswami (Trinity College, Dublin, UK), D. St Johnston (Gurdon Institute, Cambridge, UK), D. Weil (Institute André Lwoff, Villejuif, France), and B. Seraphin (Centre de Genetique Moleculaire, Centre National de la Recherche Scientifique, Gif-sur-Yvette, France). We thank Drs. S. Bhattacharyya, W. Filipowicz, E. Bertrand, and F. Rage for sharing unpublished reagents and data. We are grateful to Dr. A. Konecna, J. Vessey, and Y. Xie for critically reading this manuscript.

This article is freely available online through the J Neurosci Open Choice option.

Correspondence should be addressed to Michael Kiebler, Department of Neuronal Cell Biology, Center for Brain Research, Medical University of Vienna, 1090 Vienna, Austria. E-mail: michael.kiebler@meduniwien.ac.at.

R. Dahm's present address: Centro Nacional de Investigaciones Oncológicas, C/Melchor Fernández Almagro, 3, E-28029 Madrid, Spain.

DOI:10.1523/JNEUROSCI.0104-08.2008

Copyright $\odot 2008$ Society for Neuroscience $\quad$ 0270-6474/08/287555-08\$15.00/0 dependent remodeling of synapses and thereby to the establishment of long-term memory (Kiebler and Bassell, 2006; Sutton and Schuman, 2006). Localized mRNAs are transported in a translationally silent manner to distal dendrites in specific ribonucleoprotein particles (RNPs), termed transport RNPs. At their destinations, this translational block can be lifted by signals inducing translation (Hüttelmaier et al., 2005). At activated synapses, this is thought to occur, for example, during synaptic plasticity. Therefore, local translation of mRNAs at the activated synapse must be tightly controlled to avoid aberrant protein expression.

We have recently shown in collaboration with the Greenberg lab that small RNA-mediated silencing of transcripts plays a role at synapses of mature hippocampal neurons (Schratt et al., 2006). Because microRNAs exert their effects on mRNAs in processing bodies (P-bodies) or RNA-induced silencing complexes (RISCs) (Anderson and Kedersha, 2006; Parker and Sheth, 2007), this finding suggested that RISC and specifically P-bodies might be involved in this process. We have detected P-bodies in neurons, both in the cell body and in dendrites (Vessey et al., 2006). Furthermore, a recent study reported that P-body (DCP1a, Rck/p54/ DDX6) and transport RNP [FMRP (fragile X mental retardation protein), Staufen (Stau)] markers colocalize in dendrites of Drosophila olfactory neurons (Barbee et al., 2006), raising the possi- 
bility that P-body components contribute to the translational silencing and/or turnover of dendritically localized mRNAs. To date, however, very little is known about P-bodies in mammalian neurons (Vessey et al., 2006). We therefore decided to investigate in more detail the relationship between transport RNPs and P-bodies in mature hippocampal neurons in this study.

\section{Materials and Methods}

Neuronal culture and transient transfections. Embryonic day 17 (E17) rat hippocampal neurons were derived, cultured, and transfected as described previously (Zeitelhofer et al., 2007; Dahm et al., 2008). To minimize overexpression artifacts, we performed time series of the expression of fluorescently tagged proteins to determine the time window in which their pattern closely resembled that of the respective endogenous proteins. After 14-18 h of expression, neurons were either fixed and processed for immunocytochemistry or used for time-lapse videomicroscopy.

Constructs and protein expression. The following expression constructs were used for time-lapse videomicroscopy: red fluorescent protein (RFP)-DCP1 (Stöhr et al., 2006) and enhanced cyan fluorescent protein (ECFP)-zipcode-binding protein 1 (ZBP1) (Hüttelmaier et al., 2005) were kindly provided by Dr. Stefan Hüttelmaier (Zentrum für Angewandte Medizinische und Humanbiologische Forschung, Germany); RFP-hp54 (Rck) was kindly provided by Dr. Dominique Weil (Institute André Lwoff, Villejuif, France) (Wilczynska et al., 2005); mouse Stau1enhanced yellow fluorescent protein (EYFP) (Macchi et al., 2003a); Stau2 $62 \mathrm{kDa}$ was amplified using Pfu TaqDNA polymerase (Promega) and then cloned into the enhanced green fluorescent protein (EGFP)-C1 expression vector (Clontech/Invitrogen); EGFP-SKL was kindly provided by Dr. Andreas Hartig (University of Vienna, Vienna, Austria) (Lametschwandtner et al., 1998); DCP1-EGFP, myc-TTP, and GFP-TTP were gifts from Dr. Jens Lykke-Andersen (University of Colorado, Boulder, CO) (Lykke-Andersen and Wagner, 2005); citrine was provided by Dr. Virginie Georget (Centre National de la Recherche Scientifique, Montpellier, France) (see also Goetze et al., 2006). For the generation of antibodies, the full-length cDNA of the mouse Stau2 $62 \mathrm{kDa}$ isoform $\left(\right.$ Stau2 ${ }^{62}$ ) (Duchaîne et al., 2002) was cloned XhoI-KpnI into the pRSETb vector (Invitrogen) and into the pGEX-4T-3 vector (GE Healthcare) to obtain $\mathrm{His}_{6}$-Stau $2^{62}$ and glutathione $S$-transferase (GST)-Stau $2{ }^{62}$ recombinant proteins, respectively. For expression of $\mathrm{His}_{6}$ - or GST-tagged Barentsz (Btz) recombinant proteins, the full-length cDNA of rat Btz (GenBank accession number AF525467) was amplified from the GFPBtz construct (Macchi et al., 2003b) using a forward primer containing a BglII restriction site 5' -TTAGATCTATGGCGGACCGGCGGCGGC-3' and a reverse primer containing an $\mathrm{XhoI}$ restriction site 5'-AACTCGAGTTAACTGGAACCTCTGCTTACAAC- $3^{\prime}$, and was subcloned into the pRSETb vector (Invitrogen) and pGEX-4T-3 vector (GE Healthcare), respectively. The constructs were expressed in the Escherichia coli strain Rosetta (DE3) pLysS (Novagen). The $\mathrm{His}_{6}$-tagged fusion proteins were affinity purified under denaturing conditions using $8 \mathrm{~m}$ urea and a HISSelect HF Ni-NTA column (Sigma) and were refolded into the native conformation on the column. The following short hairpin RNA (shRNA)-encoding plasmids were used in this study: shBtz targeting rat Btz mRNA (5'-GGACCAATCTATACCCATG-3'; starting at nucleotide 1651, GenBank accession number AF525467); shStaul targeting rat Stau1 mRNA (Kanai et al., 2004); and shStau2 targeting rat Stau2 mRNA (Goetze et al., 2006). All shRNA constructs were cloned into the pSUPERIOR vector (Oligoengine) and confirmed by sequencing before use. Knockdown of the target proteins was assessed by immunocytochemistry after $3 \mathrm{~d}$ of shRNA expression.

Generation of polyclonal antibodies. To generate Stau2 and Btz polyclonal antisera, affinity-purified $\mathrm{His}_{6}$-tagged proteins were used for immunization of rabbits. Anti-Stau 2 and anti-Btz antibodies were affinity purified using GST-Stau2 ${ }^{62}$ or GST-Btz coupled to glutathione Sepharose $4 \mathrm{~B}$ beads (GE Healthcare). All antibodies used in this study were tested for specificity on Western blots via rat embryonic brain lysates (see Fig. $1 A$ ). For other antibodies used in this study, see below, Immunocytochemistry and fluorescence microscopy.
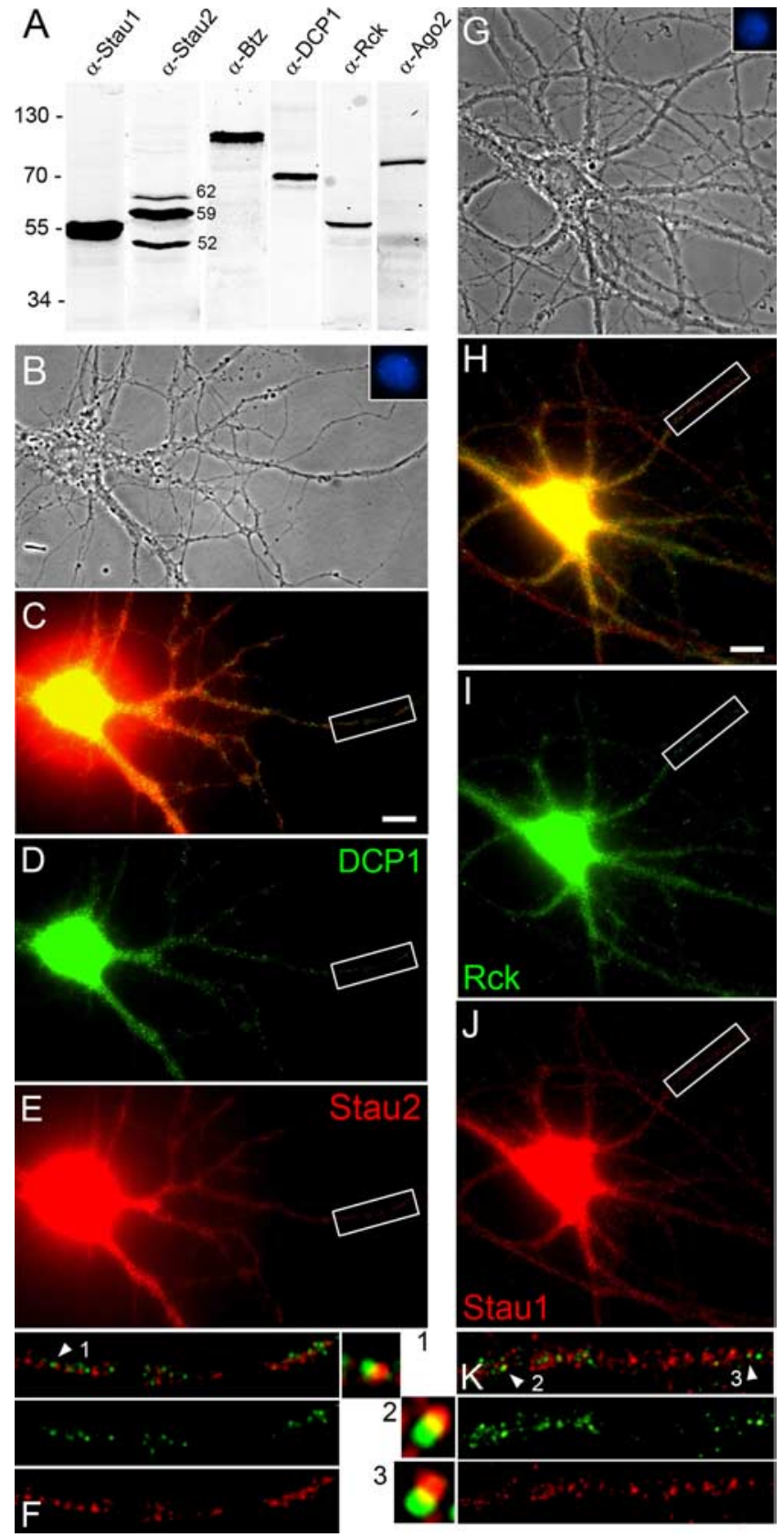

Figure 1. P-body and transport RNP markers do not colocalize in hippocampal neurons. $A$, Western blot analysis demonstrating specificity of the antibodies used in this study. All antibodies were tested on rat embryonic brain lysates. Note that the anti-Stau2 antibody recognizes the three different Stau2 isoforms (indicated by molecular weights on the right of the lane) expressed in the mammalian brain. $\boldsymbol{B}-\boldsymbol{K}$, Cultures of mature hippocampal neurons (18 DIV) were labeled with antibodies against the P-body markers DCP1 (D; in green) or Rck $(\boldsymbol{I}$; in green) and the transport RNP markers Stau2 (E; in red) or Stau1 (J; in red), respectively. $\boldsymbol{B}, \mathbf{G}$, Phase-contrast pictures of the neurons shown in $\boldsymbol{C}-\boldsymbol{E}$ and $\boldsymbol{H}-\boldsymbol{J}$, respectively, to demonstrate their integrity; insets in $\boldsymbol{B}$ and $\boldsymbol{G}$ show the corresponding DAPI-stained nuclei of those neurons. In both sets of experiments, $\mathrm{P}$-body markers show virtually no colocalization with transport RNP markers in dendrites. $\boldsymbol{C}, \boldsymbol{H}$, Merged images of Stau2 and DCP1 signals (magnified distal dendrite in $\boldsymbol{F} ; \boldsymbol{C}$ ) and of Stau1 and Rck signals (magnified distal dendrite in $\boldsymbol{K} ; \boldsymbol{H}$ ). Please note the presence of docked particles in $\boldsymbol{F}$ and $\boldsymbol{K}$ (arrowheads and magnified insets 1-3). Scale bars, $10 \mu \mathrm{m}$.

Western blotting. Western blotting was performed as described previously (Macchi et al., 2003b). Approximately 50-100 $\mu \mathrm{g}$ of total protein of E17 brain lysate was loaded per lane. Primary antibodies were used at the following dilutions: anti-Ago2, 1:100; anti-Stau1, 1:300; anti-DCP1, anti-Rck, anti-Btz, and anti-Stau2, all 1:500. For the antibodies directed 
Table 1. Quantification of data from Figure 1 and supplemental Figures 1 and 2 (available at www.jneurosci.org as supplemental material)

\begin{tabular}{|c|c|c|c|c|c|c|}
\hline Costained pairs & Stau1/DCP1 & Stau2/DCP1 & Btz/DCP1 & Stau1/Rck & Stau2/Rck & Btz/Rck \\
\hline No. of transport RNPs & $244 \pm 3.7$ & $294 \pm 17.2$ & $261 \pm 7.4$ & $269 \pm 9.4$ & $212 \pm 12$ & $229 \pm 9.7$ \\
\hline No. of P-bodies & $71 \pm 5.1$ & $109 \pm 7.1$ & $90 \pm 7.0$ & $73 \pm 2.1$ & $66 \pm 6.9$ & $65 \pm 3.9$ \\
\hline No. of colocalizations & $1.9 \pm 0.6$ & $2.8 \pm 0.8$ & $1.1 \pm 0.2$ & $1.7 \pm 0.4$ & $2.7 \pm 0.7$ & $1.4 \pm 0.7$ \\
\hline P-bodies that colocalize with RNPs & $0.8 \pm 0.3 \%$ & $0.9 \pm 0.3 \%$ & $0.4 \pm 0.1 \%$ & $0.6 \pm 0.1 \%$ & $1.3 \pm 0.3 \%$ & $0.6 \pm 0.3 \%$ \\
\hline No. of docked particles & $24 \pm 4.5$ & $33 \pm 7.2$ & $30 \pm 7.5$ & $22 \pm 4.5$ & $22 \pm 3.1$ & $26 \pm 5.1$ \\
\hline P-bodies that dock with RNPs & $34 \pm 6.3 \%$ & $30 \pm 6.6 \%$ & $33 \pm 8.3 \%$ & $30 \pm 6.2 \%$ & $33 \pm 4.7 \%$ & $40 \pm 7.8 \%$ \\
\hline
\end{tabular}

against Btz, Stau1, and Stau2, the indicated dilutions correspond to an antibody concentration of $1 \mu \mathrm{g} / \mathrm{ml}$. After incubation with primary antibodies, Western blots were probed with anti-mouse and anti-rabbit IRDYE800-conjugated secondary antibodies $(1: 10,000)$, and scanned and analyzed using the Odyssey infrared imaging system (Li-Cor Biosciences).

Pharmacological treatments. To completely silence synaptic activity, neurons were incubated overnight in $1 \mu \mathrm{M}$ TTX, $50 \mu \mathrm{M}$ APV, $100 \mu \mathrm{M}$ CNQX, and $25 \mu \mathrm{m}$ strychnine (all Sigma) as described by Vessey et al. (2006) without strychnine. To assess the effects of the glutamate stimulation on DCP1 expression and localization, neurons were treated with $10 \mu \mathrm{M}$ glutamate for $2 \mathrm{~min}$ and then incubated in NMEM-B27 for the indicated time points. Alternatively, neurons were treated with $30 \mu \mathrm{M}$ NMDA for $30 \mathrm{~min}$ or $100 \mathrm{ng} / \mathrm{ml}$ BDNF for $4 \mathrm{~h}$. The effect of oxidative stress on cultured neurons was assessed by exposure to $100 \mu \mathrm{M} \mathrm{H}_{2} \mathrm{O}_{2}$ for $4 \mathrm{~h}$. To induce a cellular stress response, neurons were incubated in medium containing $100 \mu \mathrm{M}$ arsenite (Vessey et al., 2006). After the treatments, neurons were washed and fixed immediately or incubated for the indicated time in NMEM-B27 before fixation. After these treatments, the cells were processed for immunocytochemistry.

Immunocytochemistry and fluorescence microscopy. Immunocytochemistry on mature primary hippocampal neurons in culture was performed as described previously (Goetze et al., 2006). In brief, hippocampal neurons grown on glass coverslips were washed in prewarmed $\left(37^{\circ} \mathrm{C}\right)$ modified HBSS [pH to 7.3 including $100 \mu \mathrm{g} / \mathrm{ml}$ streptomycin (Sigma)], fixed in $4 \%(\mathrm{w} / \mathrm{v})$ paraformaldehyde (Merck) for $15 \mathrm{~min}$, washed three times with PBS (Invitrogen), permeabilized with 1\% Triton X-100 (Sigma) in PBS for 5 min, and blocked with $2 \%$ fetal calf serum (PAA Laboratories), 2\% BSA (Sigma), and $0.2 \%$ fish skin gelatin (Sigma) in PBS for $30 \mathrm{~min}$. Neurons were then incubated with the respective primary antibodies for $2 \mathrm{~h}$ at room temperature, washed three times with PBS, incubated with the secondary antibodies for $45 \mathrm{~min}$ at room temperature, 4',6'-diamidino-2-phenylindole (DAPI) stained (Sigma), washed three times with PBS and one time with double-distilled $\mathrm{H}_{2} \mathrm{O}$ and mounted with $10 \%$ Mowiol (Merck), 25\% (v/v) glycerol (Sigma), and $2.5 \%(\mathrm{w} / \mathrm{v})$ DABCO antifade reagent (Sigma) in PBS. The following antibodies were used: affinity-purified rabbit polyclonal anti-Stau1 (Mallardo et al., 2003), anti-Stau2, and anti-Btz (all diluted 1:500); rabbit polyclonal anti-DCP1a (1:1000; kindly provided by Dr. Mani Ramaswami, Trinity College, Dublin, UK) (Barbee et al., 2006); rabbit polyclonal anti-Rck (1:1000; MBL International); mouse polyclonal antiDCP1a (1:200), mouse monoclonal anti-DDX6/Rck (1:200), and mouse monoclonal anti-Ago2 (1:200; all from Abnova); mouse monoclonal anti-Tau (1:250; Millipore Bioscience Research Reagents); mouse monoclonal anti-PSD-95 (1:2000; Sigma); and mouse monoclonal anti-TIA-1 (T-cell intracellular antigen 1)-related protein (anti-TIAR; 1:100; Transduction Laboratories). Cy3- and Alexa 488-coupled goat anti-mouse and goat anti-rabbit IgG secondary antibodies (dilution, 1:2000) were used for detection (Dianova). All antibodies were diluted in the indicated concentrations in $10 \%$ blocking solution. Mitochondria were labeled by incubating neuronal cultures with a dilution 1:10,000 MitoTracker Green (Invitrogen) in NMEM for $30 \mathrm{~min}$. Fluorescence and phasecontrast images were acquired with an Axiovert 200M microscope (Zeiss). Images were acquired using a $40 \times$ Plan-Neofluor [numerical aperture (NA), 1.3] or a $63 \times(\mathrm{NA}, 1.4)$ Plan-Apochromat oil-immersion objective (both Zeiss), a Coolsnap HQ CCD camera (Visitron/Roper Scientific), and the MetaMorph 6.3 imaging software package (Universal Imaging/Visitron) and assembled with Adobe Photoshop 7.0. Images were not modified other than adjustments of levels, brightness, contrast, and magnification.

Time-lapse videomicroscopy. For live imaging, primary hippocampal neurons on glass coverslips were transferred into HBSS and placed into an imaging chamber mounted onto an Axiovert 200M microscope (Zeiss). Images were acquired at a frame rate of 1 image per 5-20 $\mathrm{s}$ as indicated using a $40 \times$ or a $63 \times$ oil-immersion objective. Time-lapse videos were acquired using the MetaMorph 6.3 software package (Universal Imaging/Visitron) and movies generated with QuickTime PlayerPro 7.0.3 (Apple Computer). Images were not modified other than adjustments of levels, brightness, contrast, and magnification.

Deconvolution and confocal microscopy. For deconvolution microscopy, images were acquired on a DeltaVision Spectris Restoration Microscopy system (Applied Precision) equipped with an IX71 microscope (Olympus), a 100×/1.4 PlanApo oil-immersion objective (Olympus), and a CoolSNAP HQ camera (Roper Scientific). Optical sections were acquired with a spacing of $150 \mathrm{~nm}$. Images were subsequently deconvolved with softWoRx (Applied Precision). Confocal laser-scanning microscopy was performed with an LSM510 (Zeiss), and 40×/1.3 PlanNeofluar or $63 \times / 1.4$ PlanApochromat oil-immersion objectives (Zeiss). Live LSM imaging was performed with an LSM5Duo microscope (Zeiss) and a $40 \times / 1.3$ PlanNeofluar oil-immersion objective.

\section{Results}

To investigate whether transport RNPs colocalize with P-body components in mammalian neurons, we immunostained primary cultures of mature hippocampal neurons $[15 \mathrm{~d}$ in vitro (DIV)] with antibodies against P-body and RNP markers. To detect transport RNPs involved in dendritic mRNA localization (termed transport RNPs in the remainder of the manuscript), we generated mono-specific, affinity-purified antibodies directed against rat Stau1, mouse Stau2, and rat Btz as markers (Fig. 1A; supplemental Fig. 3, available at www.jneurosci.org as supplemental material) (Mallardo et al., 2003) (D. Karra and M. A. Kiebler, unpublished results). The RNA-binding proteins Btz and Stau are most consistently associated with RNA transport in Drosophila (Martin et al., 2003; St Johnston, 2005) and move bidirectionally in RNPs in dendrites of mammalian neurons (Macchi et al., 2003b; Goetze et al., 2006) (this study). Moreover, we used the zipcode-binding protein 1 (ZBP1), a protein shown to be essential for mRNA localization and translational control in fibroblasts (Lapidus et al., 2007) and neuronal cells (Hüttelmaier et al., 2005), as a marker for transport RNPs. For P-body markers, we chose the helicase Rck (DDX6/p54, mammalian homolog of Drosophila Me31b and of yeast Dhh1p) and the decapping enzyme DCP1 (Barbee et al., 2006).

All transport RNP and P-body markers used show virtually no colocalization in proximal and distal dendrites (Fig. 1, Table 1; supplemental Figs. 1, 2, 5, Table 1, available at www.jneurosci.org as supplemental material), indicating that these two complexes are distinct in their molecular composition. To test whether other proteins involved in mRNA translational control and/or degradation are also specifically excluded from transport RNPs, we performed colocalization experiments with Ago2 (Höck et al., 2007) and Staul (data not shown). These experiments confirm 
our results of strict separation between transport RNPs and other RNA granules. In contrast, different antibodies against the two P-body markers used in this study (for their specificity, see Fig. $1 A$ ) consistently show complete colocalization (supplemental Fig. 4, available at www.jneurosci.org as supplemental material), confirming that we are able to detect substantial colocalization events. The absence of colocalization of P-body and transport RNP markers is further corroborated by immunoprecipitation experiments using mono-specific, affinity-purified Staul and Stau2 antibodies, which failed to precipitate DCP1 (data not shown) (Karra and Kiebler, unpublished results). A surprising finding, however, was that frequently transport RNPs and P-bodies are located next to each other, termed docked particles (Fig. 1 F, K, inset, Table 1; supplemental Fig. 5, Table 1, available at www.jneurosci.org as supplemental material).

To address the question of whether P-bodies and transport RNPs might transiently be transported within the same dendritic RNPs or whether they transiently interact, we performed timelapse videomicroscopy (Dahm et al., 2008). To visualize transport RNP and P-bodies in living hippocampal neurons, we cotransfected fluorescently tagged constructs encoding either RFPDCP1 or Rck-RFP together with EGFP-Stau2 or Stau1-EYFP. Control experiments using those constructs show the same localization pattern compared with the endogenous transport RNP and P-body markers (supplemental Figs. $4 K-L, 6 B$, available at www.jneurosci.org as supplemental material).

Live videomicroscopy analyses of neurons transfected with RFP-DCP1 showed that dendritic P-bodies are very motile structures with velocities similar to those observed for transport RNPs (Köhrmann et al., 1999). Interestingly, we observe that P-bodies, when motile, always move independently of transport RNPs as visualized by cotransfection of neurons with Stau1-EYFP, EGFPStau2, and ECFP-ZBP1, respectively (Fig. 2A-F; supplemental Figs. 7, 8, Videos 1-3, available at www.jneurosci.org as supplemental material). As previously reported for transport RNPs (Köhrmann et al., 1999), $50 \%$ of motile P-bodies move unidirectionally, with the other half moving in a bidirectional manner with rapid switches in direction (Fig. $2 G$ ). From those moving unidirectionally, approximately the same proportion moves in an anterograde and retrograde manner, respectively (Fig. 2G). This suggests that there is no preferential direction for P-body movement. Interestingly, incubating cultured neurons in medium containing $60 \mu \mathrm{M}$ nocodazole affected motility, but not P-body numbers in dendrites (data not shown), suggesting that they move along dendritic microtubules. Together, our finding that P-bodies can move from the soma into dendrites (Fig. $2 A-C)$ raises the possibility that the dendritic pool of P-bodies is, at least in part, derived from somatic P-bodies.

Our analyses of living neurons revealed that the observed docked particles are not an artifact of the immunocytochemistry procedure or of the high densities observed for endogenous transport RNPs (see also supplemental Fig. 5, available at www. jneurosci.org as supplemental material), but that docking appears to be an active and dynamic process. A large fraction of P-bodies (55 and 46\%, respectively, for Stau1/DCP1 and Stau2/ DCP1) (Fig. 3, Table 1; supplemental Table 1, Fig. 8, Videos 5-9, available at www.jneurosci.org as supplemental material) are docked to transport RNPs; i.e., the two particle types are immediately adjacent to each other and remain that way for at least 1 min (Fig. 3). We found docked particles to transiently disassociate and reassociate with varying time courses (Fig. $3 C, D, K$ ). Importantly, this docking occurs both in proximal and distal regions of dendrites [see boxed areas in Fig. $3 A, B$ and supplemental Fig.
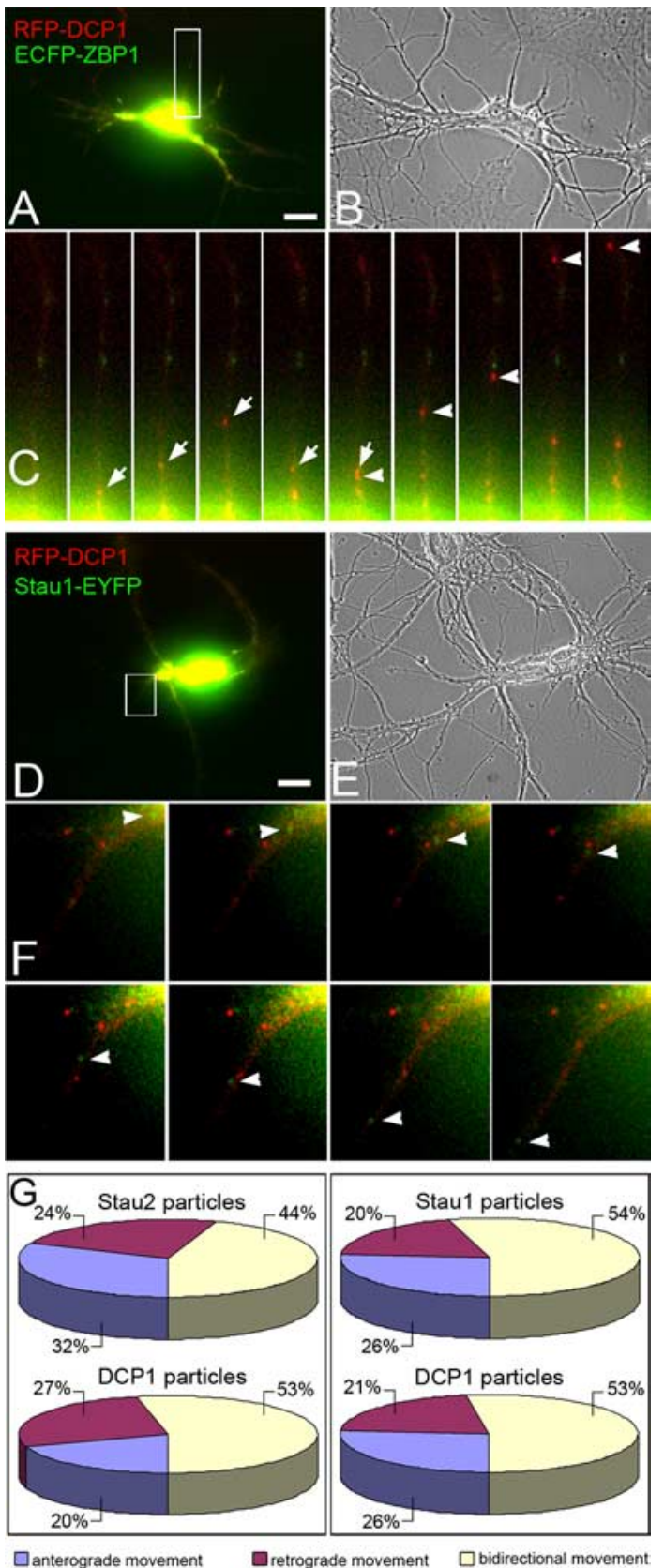

Figure 2. Fluorescently labeled P-bodies and transport RNPs move independently in dendrites. $\boldsymbol{A}-\boldsymbol{F}$, Mature hippocampal neurons (16 DIV) were cotransfected with expression constructs encoding RFP-DCP1 (in red) and ECFP-ZBP1 ( $\boldsymbol{A}-\boldsymbol{C}$; ; seudocolored in green) or RFP-DCP1 (in red) and Stau1-EYFP ( $D-F ;$ pseudocolored in green), respectively. After $14 \mathrm{~h}$ of expression, the respective $P$-body and transport RNP markers were visualized by time-lapse videomicroscopy. $\boldsymbol{B}, \boldsymbol{E}$, After live imaging, phase-contrast images were acquired to assess the integrity of the neurons. $\boldsymbol{C}$, Higher magnification of a dendrite (boxed region in $\boldsymbol{A}$ ) in which DCP1 particles (in red; arrows and arrowheads) move independently of the transport RNP marker ZBP1 (pseudocolored in green) in a bidirectional manner. The boxed region in $\boldsymbol{D}$ shows that Stau1 ( $\boldsymbol{F}$, arrowheads) moves independently from DCP1 into distal dendrites. See supplemental Videos 1 and 2 (available at www.jneurosci.org as supplemental material). G, Directionality of DCP1, Stau1, and Stau2 particle movements in dendrites of mature hippocampal neurons. Particles were classified as moving unidirectionally if the direction of their movement did not change throughout the examination period. Scale bars, $10 \mu \mathrm{m}$.

8 (available at www.jneurosci.org as supplemental material)], including in regions where the particle density is very low. This observation precludes the possibility that docking is an artifact of particle density. Moreover, P-bodies show no tendency to dock 


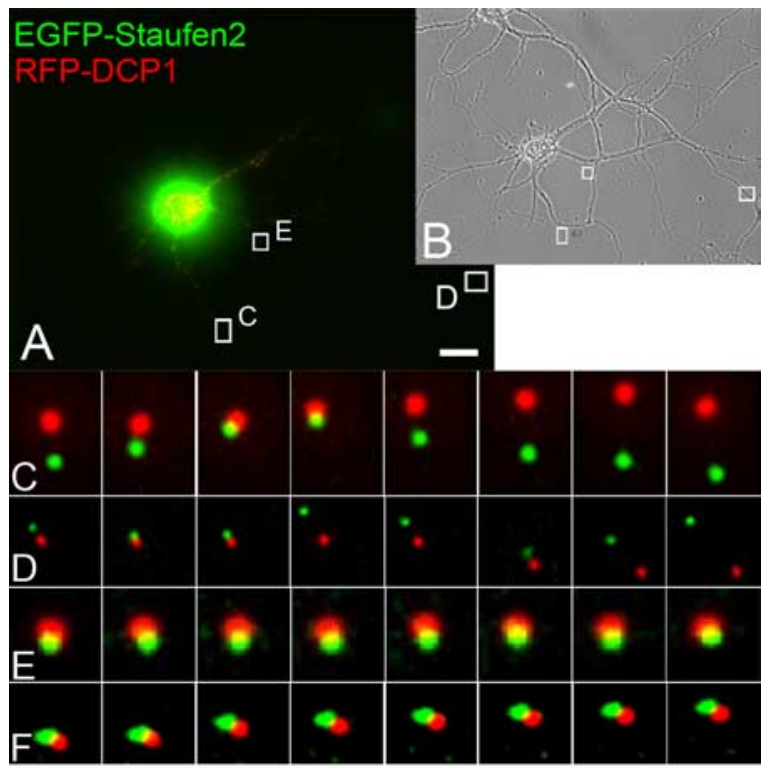

G Analysis of transport RNP \& P body populations
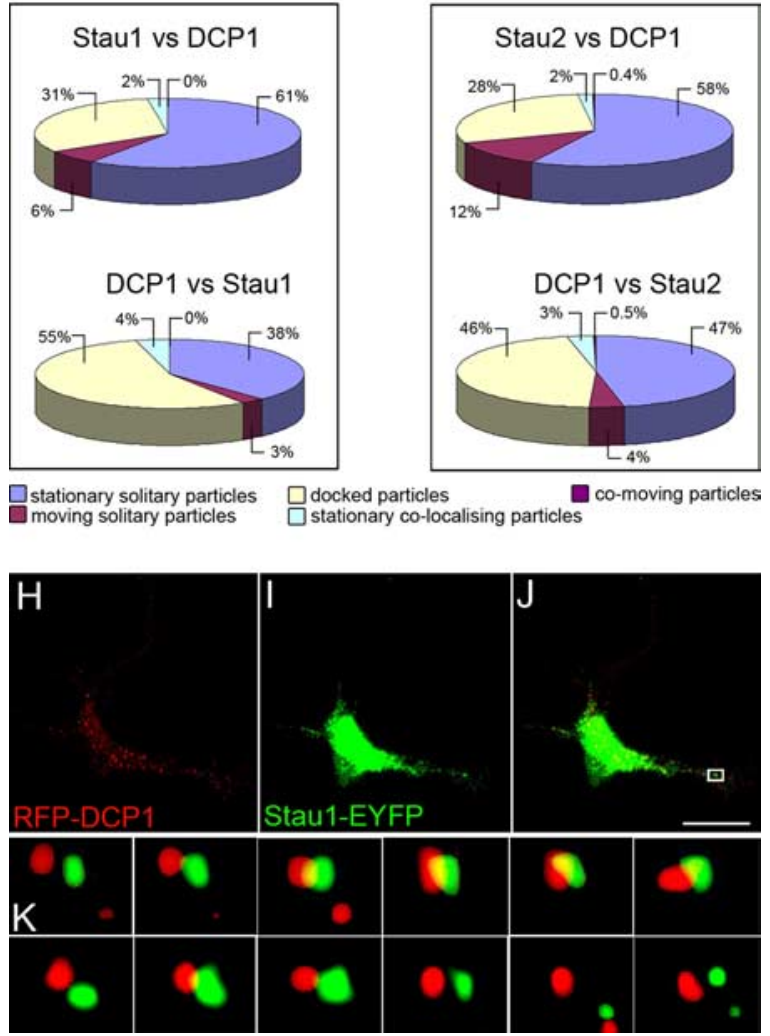

Figure 3. P-bodies and transport RNPs are physically distinct particles that temporarily dock in hippocampal neurons. Mature hippocampal neurons (15 DIV) were cotransfected with expression constructs encoding RFP-DCP1 and EGFP-Stau2. $\boldsymbol{A}$, After $14 \mathrm{~h}$ of expression, both P-body and transport RNP markers were visualized by time-lapse videomicroscopy (supplemental Video 4, available at www.jneurosci.org as supplemental material). Scale bar, $10 \mu \mathrm{m}$. $\boldsymbol{B}$, After imaging, a phase-contrast image was acquired to assess the integrity of the neuron. $\boldsymbol{C}-\boldsymbol{E}$, Higher magnifications of the boxed regions in $\boldsymbol{A}$. $\boldsymbol{C}, \boldsymbol{D}$, Independent movement of $\mathrm{P}$-body and transport RNP markers interrupted by transient docking ( $>5 \mathrm{~min}$ ). $\boldsymbol{E}$, Particles docked constantly over $34 \mathrm{~min}$ (see also supplemental Videos 5-7, available at www.jneurosci.org as supplemental material). $\boldsymbol{F}$, Enlargement from a neuron (supplemental Fig. 6, available at www.jneurosci.org as supplemental material) transfected with Stau1-EYFP (green) and RFPDCP1 (red), showing particles constantly docked for 17 min (see also supplemental Video 8, available at www.jneurosci.org as supplemental material). G, Analysis of transport RNP markers (Stau1-EYFP and EGFP-Stau2) compared with the P-body marker DCP1 in dendrites. Note with other intracellular organelles, such as mitochondria and peroxisomes (supplemental Fig. 9, Videos 10, 11, available at www.jneurosci.org as supplemental material), further supporting the hypothesis that docking of P-bodies and transport RNPs is a specific event. Interestingly, a recent study reported a similar phenomenon for stress granules and P-bodies in mammalian cell culture cells (Kedersha et al., 2005). We confirm this finding with our observation of docked, but not colocalizing, DCP1-positive P-bodies and TIAR-positive stress granules in primary rat hippocampal neurons (supplemental Fig. $4 P-R$, available at www. jneurosci.org as supplemental material).

A thorough quantification of P-body dynamics revealed that 41 and $51 \%$, respectively, of the particles remain strictly separated from transport RNPs, i.e., neither colocalizing nor docking, over the entire duration of the time-lapse experiment (Fig. $3 G$ ). Of these, only 3 and $4 \%$, respectively, in each neuron are motile. The majority are stationary with the exception of short-range oscillatory movements. P-bodies in a docked configuration are generally stationary, showing at most short-range movement on the order of a few micrometers. Only rarely (4 and 3\% of cases, respectively), P-body and transport RNP markers appear to actually colocalize. In this case, the signals for both particles are approximately of the same size and shape and largely overlap, and in very few cases $(<0.5 \%)$, P-body and transport RNP markers appear to move together within the same particles in distal dendrites. These findings strongly indicate that P-bodies and transport RNPs are molecularly distinct particles that can transiently associate. The nature and purpose of this association is at present unknown.

Because P-bodies have been suggested to represent sites of mRNA storage that repress the translation of transcripts in a reversible manner (Parker and Sheth, 2007), we investigated whether chemical activation of synapses, an experimental model to induce synaptic plasticity in cultured neurons, affects localization of P-bodies in dendrites. We stimulated neurons with glutamate (Vessey et al., 2006), BDNF (Yoshii and Constantine-Paton, 2007), or NMDA (Martin et al., 2007), respectively. To inhibit neuronal activity, we silenced neurons by incubation in a mixture containing TTX, strychnine, APV, and CNQX (Vessey et al., 2006). After these treatments, we assessed the distribution of P-bodies and transport RNPs via antibody staining (Figs. 4, 5). Interestingly, chemical stimulation of hippocampal neurons with glutamate or NMDA leads to a substantial decrease (up to 70\%) in DCP1-containing dendritic P-bodies (Fig. 4B, C,F) compared with untreated neurons (Fig. $4 A$ ). Chemical stimulation with BDNF leads to a substantial decrease in the number of DCP1-, Rck-, and Ago2-containing P-bodies, respectively (Fig. 5). In contrast, the distribution of P-bodies is unaffected by silencing of neuronal activity (Fig. 4G). Similarly, the numbers and pattern of dendritic $\mathrm{P}$-bodies remain unchanged when neurons are exposed to oxidative stress $\left(\mathrm{H}_{2} \mathrm{O}_{2}\right)$ (Fig. $\left.4 \mathrm{H}\right)$ or after incubation with

$\leftarrow$

that, as observed for endogenous transport RNP and P-body markers (Fig. 1), there is virtually no colocalization observed between Staufen proteins and DCP1. Importantly, however, a significant proportion of transport RNPs and P-bodies are found in close apposition to each other ("docking"). $\boldsymbol{H}-\boldsymbol{K}$, Videomicroscopy of living neurons by confocal microscopy confirms the dynamic nature of docking between transport RNPs and P-bodies. $\boldsymbol{H}, \boldsymbol{I}$, Cultures of mature hippocampal neurons (14 DIV) coexpressing RFP-DCP1 (red; $\boldsymbol{H}$ ) and Stau1-EYFP (green; $\boldsymbol{I}) . \boldsymbol{J}$, Merged fluorescence images. Several images taken in the region indicated by a box in $J$ are shown at higher magnification in $\boldsymbol{K}$. The sequence shows the behavior of the particles over a period of $25 \mathrm{~min}$. Note that the P-bodies and transport RNPs temporarily dock over periods of $\sim 8$ and $4 \mathrm{~min}$, respectively. Scale bar, $20 \mu \mathrm{m}$. 

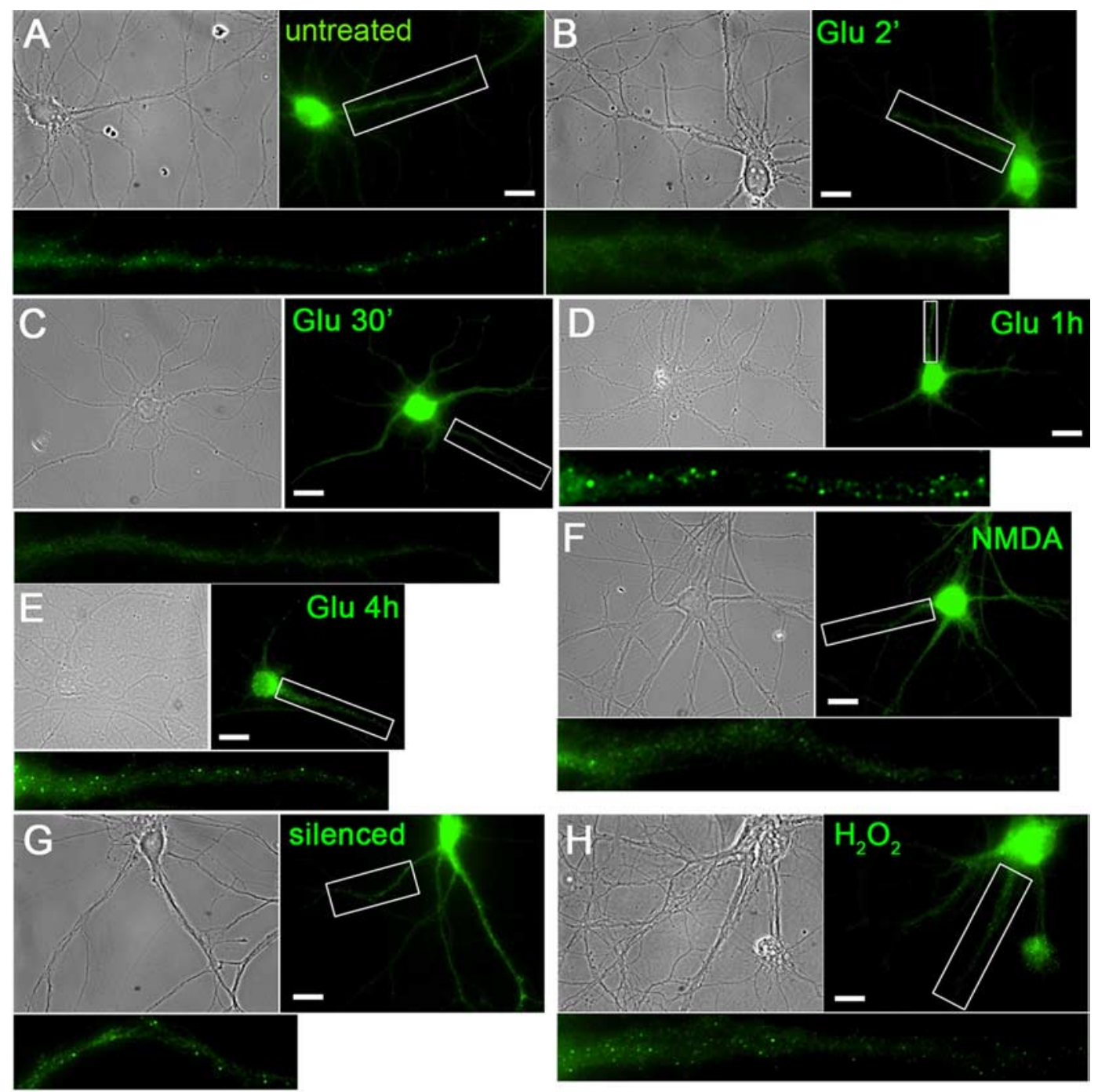

\begin{tabular}{|c|c|c|c|c|c|c|c|c|}
\hline & Untreated & $\begin{array}{l}\text { Glutamate } \\
2+0 \text { min }\end{array}$ & $\begin{array}{l}\text { Glutamate } \\
2+15 \text { min }\end{array}$ & $\begin{array}{l}\text { Glutamate } \\
2+30 \text { min }\end{array}$ & $\begin{array}{l}\text { Glutamate } \\
2 \mathrm{~min}+1 \mathrm{~h}\end{array}$ & $\begin{array}{l}\text { Glutamate } \\
2 \text { min }+4 \text { h }\end{array}$ & $\begin{array}{l}\text { BDNF } \\
4 \mathrm{~h}\end{array}$ & $\begin{array}{l}\text { NMDA } \\
30 \text { min }\end{array}$ \\
\hline $\begin{array}{l}\# \text { of all } \\
\text { P-bodies }\end{array}$ & $61 \pm 8.0$ & $37 \pm 12.7$ & $19 \pm 6.2$ & $22 \pm 8.3$ & $61 \pm 5.6$ & $65 \pm 17.0$ & $20 \pm 9.3$ & $21 \pm 7.6$ \\
\hline $\begin{array}{l}\text { \# of large } \\
\text { P-bodies }\end{array}$ & $32 \pm 5.5$ & $12 \pm 4.6$ & $6 \pm 3.7$ & $8 \pm 5.2$ & $25 \pm 5.9$ & $24 \pm 6.9$ & $15 \pm 8.3$ & $4 \pm 2.9$ \\
\hline $\begin{array}{l}\text { \# of small } \\
\text { P-bodies }\end{array}$ & $29 \pm 8.0$ & $25 \pm 9.6$ & $13 \pm 4.3$ & $14 \pm 4.6$ & $36 \pm 5.9$ & $41 \pm 11.7$ & $5 \pm 2.2$ & $17 \pm 7.7$ \\
\hline $\begin{array}{l}\text { Reduction in } \\
\text { number of all } \\
\text { P-bodies }\end{array}$ & $0 \% \pm 13 \%$ & $-39 \% \pm 21 \%$ & $-69 \% \pm 10 \%$ & $-64 \% \pm 14 \%$ & $0 \% \pm 9 \%$ & $+6 \% \pm 28 \%$ & $\begin{array}{c}-67 \% \\
\pm \\
15 \%\end{array}$ & $\begin{array}{c}-66 \% \\
\pm \\
12 \%\end{array}$ \\
\hline
\end{tabular}

Figure 4. Chemical stimulation of mature hippocampal neurons disassembles dendritic $P$-bodies. $\boldsymbol{A}-\boldsymbol{H}$, Mature hippocampal neurons (18 DIV) were mock treated $(\boldsymbol{A})$, stimulated with glutamate $(\boldsymbol{B}-\boldsymbol{E})$ for $2 \mathrm{~min}$ and incubated for the indicated time intervals, stimulated with NMDA $(\boldsymbol{F})$ for $30 \mathrm{~min}$, silenced overnight with TTX, CNQX, D-APV, and strychnine $(\boldsymbol{G})$, or exposed to oxidative stress with $\mathrm{H}_{2} \mathrm{O}_{2}(\boldsymbol{H})$ for $4 \mathrm{~h}$. After treatment, neurons were stained for the P-body marker DCP1. Insets show the boxed regions in $\boldsymbol{A}-\boldsymbol{H}$. Whereas silencing and oxidative stress $(\boldsymbol{G}, \boldsymbol{H})$ do not affect dendritic P-bodies, stimulation of neurons with glutamate $(\boldsymbol{B}, \boldsymbol{C})$ leads initially to a significant reduction in the number of dendritic $P$-bodies. $\boldsymbol{D}, \boldsymbol{E}$, After longer periods of time ( $>30$ min) after the glutamate pulse, the number of dendriticP-bodies recovers to basiclevels. Scalebars, $10 \mu \mathrm{m}$. Bottom (table), Quantification of dendritic P-body numbers in hippocampal neurons after neuronal stimulation with glutamate, NMDA, or BDNF. For the quantification, five (glutamate) and three (NMDA, BDNF) independent experiments (10 neurons each), respectively, were evaluated in a blind manner. See also Figure 5.

arsenite (data not shown). This indicates that P-body disassembly might occur specifically after synaptic activation and is likely not an artifact of cellular stress. Notably, the numbers and distribution of transport RNPs are not affected by neuronal stimulation, silencing, or exposure to stress (data not shown). There are, however, also alternative interpretations for the apparent reduc- tion in the numbers of DCP1- and Rck-positive particles than an activity-driven dissociation of P-bodies. For one, P-bodies might undergo high turnover and their reassembly and/or de novo formation might be altered by neuronal activity. Unpublished data from FRAP experiments suggest, however, that dendritic P-bodies may be significantly more stable structures than their 

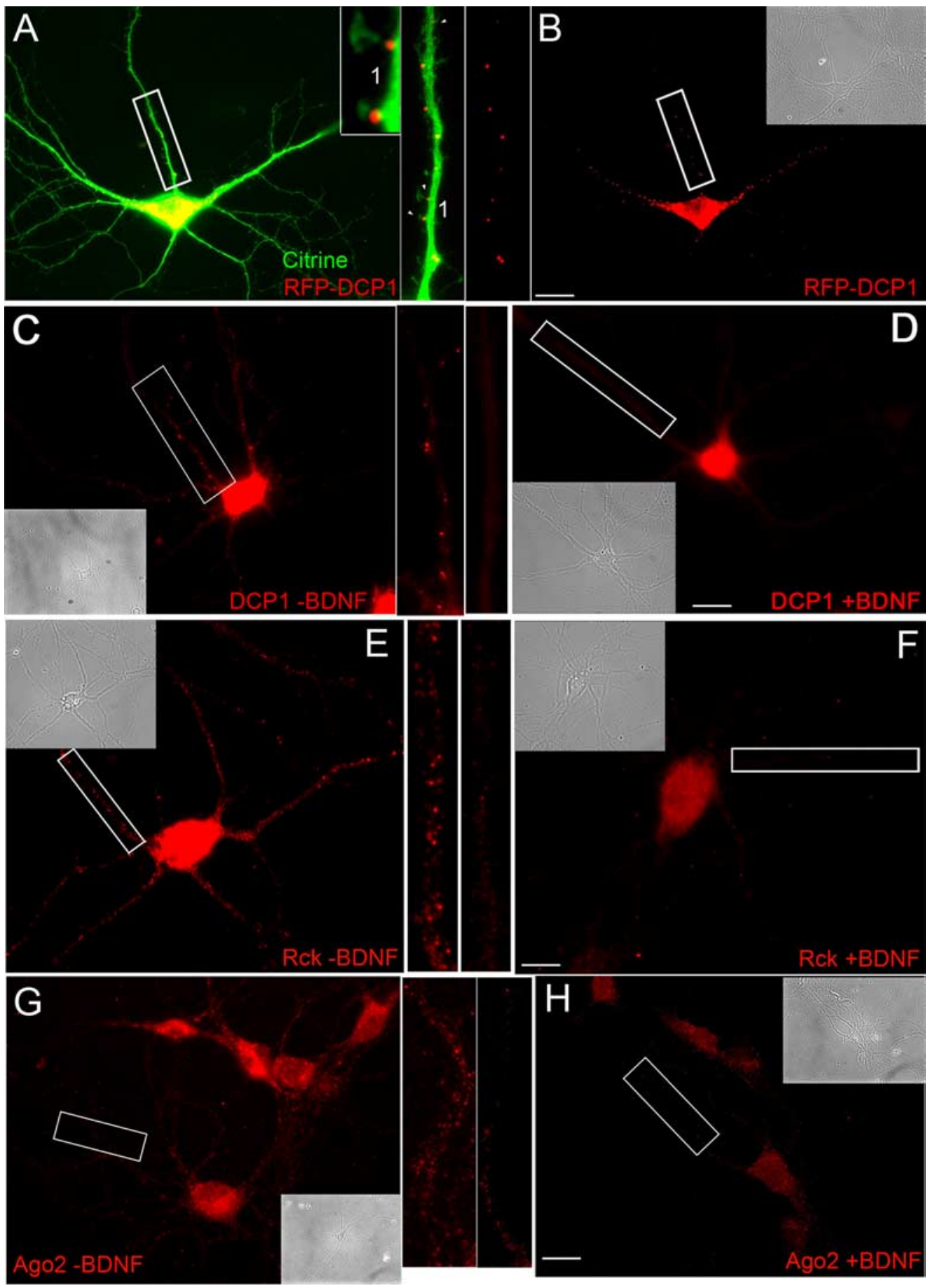

Figure 5. P-bodies localize at the bases of dendritic spines, and BDNF stimulation disassembles dendritic P-bodies. $A, B$, Mature (14 DIV) hippocampal neurons were cotransfected with expression vectors encoding RFP-DCP1 ( $\boldsymbol{B}$; red) to label P-bodies and citrine ( $\boldsymbol{A}$; merged image) to visualize the morphology of the neuron. Enlargements of part of a dendrite (boxed regions in $\boldsymbol{A}, \boldsymbol{B})$ show numerous P-bodies located at the bases of dendritic spines (arrowheads). Thirty percent of total DCP1 particles are located at the base of spines (30 neurons quantified; $25 \pm 11.9$ total DCP1 particles per cell; $7.5 \pm 4.3 \mathrm{Dcp} 1$ particles in spines). Mature hippocampal neurons (15 DIV) were either mock treated $(\boldsymbol{C}, \boldsymbol{E}, \boldsymbol{G})$ or stimulated with $100 \mathrm{ng} / \mathrm{ml} \mathrm{BDNF}(\boldsymbol{D}, \boldsymbol{F}, \boldsymbol{H})$ for $4 \mathrm{~h}$. After treatment, neurons were stained for the P-body markers DCP1, Rck, and Ago2, respectively. Insets show the boxed regions in $\mathbf{C}-\boldsymbol{H}$. Interestingly, BDNF stimulation of neurons led to a significant reduction in the number of dendritic $\mathbf{P}$-bodies as detected with either of the three markers. The insets in $\boldsymbol{B}-\boldsymbol{H}$ show the respective phase-contrast images of the neurons. Please note that to ensure comparability between the fluorescent signals from neurons after stimulation with BDNF and mock treatment, respectively, all images were acquired with the same settings and processed in the identical way. Scale bars, $20 \mu \mathrm{m}$.

counterparts in HeLa cells (F. Rage, E. Bertrand, and W. Filipowicz, personal communication). Alternatively, only individual proteins, such as DCP1 (Fig. 4) and Rck (Fig. 5), might be lost from P-bodies, which remain otherwise intact. This latter point can only be clarified as more bona fide P-body markers are identified.
Together, our data suggest that P-bodies, especially the larger particles (Fig. 4A), disassemble after synaptic stimulation (Figs. $4 B, C, F, 5 D, F, H)$. After longer periods of time (>30 min) after the brief exposure to glutamate, we observed a recovery in the numbers of dendritic P-bodies to basic levels (Fig. $4 D, E$ ). In this context, it is interesting to note that P-bodies are found at the 
bases of dendritic spines in hippocampal neurons (Fig. 5A,B). These results lend support to the exciting hypothesis that mRNAs stored in dendritic P-bodies might be released and possibly translated when synapses become activated. A recent study provided interesting evidence that P-bodies might function as a reservoir that sequesters mRNAs containing AU-rich elements or microRNA binding sites from polysomes, thereby silencing them (Franks and Lykke-Andersen, 2007). Furthermore, there is evidence that dendritic P-bodies contain both mRNAs that have microRNA binding sites in their $3^{\prime}$ - untranslated regions and microRNAs (Rage, Bertrand, and Filipowicz, personal communication). These observations suggest a role of dendritic P-bodies in the translational control of dendritically localized mRNAs.

\section{Discussion}

Our findings that P-bodies and transport RNPs are molecularly distinct are not in line with observations by Barbee et al. (2006) in Drosophila peripheral neurons, in which a very large degree of colocalization between Staufen and DCP1 was reported. This disparity may reflect a genuine difference between mammalian and insect neurons. Alternatively, certain conditions may exist under which the two particle types might also fuse in mammalian neurons. A better understanding of the function of P-bodies in dendrites and in particular of the functional relevance of their docking with transport RNPs may help contribute to resolving this apparent conflict.

\section{References}

Anderson P, Kedersha N (2006) RNA granules. J Cell Biol 172:803-808.

Barbee SA, Estes PS, Cziko AM, Hillebrand J, Luedeman RA, Coller JM, Johnson N, Howlett IC, Geng C, Ueda R, Brand AH, Newbury SF, Wilhelm JE, Levine RB, Nakamura A, Parker R, Ramaswami M (2006) Staufen- and FMRP-containing neuronal RNPs are structurally and functionally related to somatic P bodies. Neuron 52:997-1009.

Bramham CR, Wells DG (2007) Dendritic mRNA: transport, translation and function. Nat Rev Neurosci 8:776-789.

Dahm R, Zeitelhofer M, Götze B, Kiebler MA, Macchi P (2008) Visualizing mRNA localization and local protein translation in neurons. Methods Cell Biol 85:293-327.

Duchaîne TF, Hemraj I, Furic L, Deitinghoff A, Kiebler MA, DesGroseillers L (2002) Staufen2 isoforms localize to the somatodendritic domain of neurons and interact with different organelles. J Cell Sci 115:3285-3295.

Franks TM, Lykke-Andersen J (2007) TTP and BRF proteins nucleate processing body formation to silence mRNAs with AU-rich elements. Genes Dev 21:719-735.

Goetze B, Tuebing F, Xie Y, Dorostkar MM, Thomas S, Pehl U, Boehm S, Macchi P, Kiebler MA (2006) The brain-specific double-stranded RNAbinding protein Staufen2 is required for dendritic spine morphogenesis. J Cell Biol 172:221-231.

Höck J, Weinmann L, Ender C, Rüdel S, Kremmer E, Raabe M, Urlaub H, Meister G (2007) Proteomic and functional analysis of Argonautecontaining mRNA-protein complexes in human cells. EMBO Rep 8:1052-1060.

Hüttelmaier S, Zenklusen D, Lederer M, Dictenberg J, Lorenz M, Meng X, Bassell GJ, Condeelis J, Singer RH (2005) Spatial regulation of betaactin translation by Src-dependent phosphorylation of ZBP1. Nature 438:512-515.

Kanai Y, Dohmae N, Hirokawa N (2004) Kinesin transports RNA: isolation and characterization of an RNA-transporting granule. Neuron 43:513-525.

Kedersha N, Stoecklin G, Ayodele M, Yacono P, Lykke-Andersen J, Fritzler
MJ, Scheuner D, Kaufman RJ, Golan DE, Anderson P (2005) Stress granules and processing bodies are dynamically linked sites of mRNP remodeling. J Cell Biol 169:871-884.

Kiebler MA, Bassell GJ (2006) Neuronal RNA granules: movers and makers. Neuron 51:685-690.

Köhrmann M, Luo M, Kaether C, DesGroseillers L, Dotti CG, Kiebler MA (1999) Microtubule-dependent recruitment of Staufen-green fluorescent protein into large RNA-containing granules and subsequent dendritic transport in living hippocampal neurons. Mol Biol Cell 10:2945-2953.

Lametschwandtner G, Brocard C, Fransen M, Van Veldhoven P, Berger J, Hartig A (1998) The difference in recognition of terminal tripeptides as peroxisomal targeting signal 1 between yeast and human is due to different affinities of their receptor Pex $5 \mathrm{p}$ to the cognate signal and to residues adjacent to it. J Biol Chem 273:33635-33643.

Lapidus K, Wyckoff J, Mouneimne G, Lorenz M, Soon L, Condeelis JS, Singer RH (2007) ZBP1 enhances cell polarity and reduces chemotaxis. J Cell Sci 120:3173-3178.

Lykke-Andersen J, Wagner E (2005) Recruitment and activation of mRNA decay enzymes by two ARE-mediated decay activation domains in the proteins TTP and BRF-1. Genes Dev 19:351-361.

Macchi P, Hemraj I, Goetze B, Grunewald B, Mallardo M, Kiebler MA (2003a) A GFP-based system to uncouple mRNA transport from translation in a single living neuron. Mol Biol Cell 14:1570-1582.

Macchi P, Kroening S, Palacios IM, Baldassa S, Grunewald B, Ambrosino C, Goetze B, Lupas A, St Johnston D, Kiebler M (2003b) Barentsz, a new component of the Staufen-containing ribonucleoprotein particles in mammalian cells, interacts with Staufen in an RNA-dependent manner. J Neurosci 23:5778-5788.

Mallardo M, Deitinghoff A, Müller J, Goetze B, Macchi P, Peters C, Kiebler MA (2003) Isolation and characterization of Staufen-containing ribonucleoprotein particles from rat brain. Proc Natl Acad Sci U S A 100:2100-2105.

Martin S, Nishimune A, Mellor JR, Henley JM (2007) SUMOylation regulates kainate-receptor-mediated synaptic transmission. Nature 447:321-325.

Martin SG, Leclerc V, Smith-Litière K, St Johnston D (2003) The identification of novel genes required for Drosophila anteroposterior axis formation in a germline clone screen using GFP-Staufen. Development 130:4201-4215.

Parker R, Sheth U (2007) P bodies and the control of mRNA translation and degradation. Mol Cell 25:635-646.

Schratt GM, Tuebing F, Nigh EA, Kane CG, Sabatini ME, Kiebler M, Greenberg ME (2006) A brain-specific microRNA regulates dendritic spine development. Nature 439:283-289.

St Johnston D (2005) Moving messages: the intracellular localization of mRNAs. Nat Rev Mol Cell Biol 6:363-375.

Stöhr N, Lederer M, Reinke C, Meyer S, Hatzfeld M, Singer RH, Hüttelmaier S (2006) ZBP1 regulates mRNA stability during cellular stress. J Cell Biol 175:527-534.

Sutton MA, Schuman EM (2006) Dendritic protein synthesis, synaptic plasticity, and memory. Cell 127:49-58.

Vessey JP, Vaccani A, Xie Y, Dahm R, Karra D, Kiebler MA, Macchi P (2006) Dendritic localization of the translational repressor Pumilio 2 and its contribution to dendritic stress granules. J Neurosci 26:6496-6508.

Wilczynska A, Aigueperse C, Kress M, Dautry F, Weil D (2005) The translational regulator CPEB1 provides a link between dcp1 bodies and stress granules. J Cell Sci 118:981-992.

Yoshii A, Constantine-Paton M (2007) BDNF induces transport of PSD-95 to dendrites through PI3K-AKT signaling after NMDA receptor activation. Nat Neurosci 10:702-711.

Zeitelhofer M, Vessey JP, Xie Y, Tübing F, Thomas S, Kiebler M, Dahm R (2007) High-efficiency transfection of mammalian neurons via nucleofection. Nat Protoc 2:1692-1704. 